\title{
Chiral Recognition of Amino Acids by Magnetoelectrodeposited Cu Film Electrodes
}

\author{
Iwao Mogi and Kazuo Watanabe \\ Institute for Materials Research, Tohoku University, Katahira, Aoba-ku, Sendai 980-8577, Japan \\ Correspondence should be addressed to Iwao Mogi, mogi@imr.tohoku.ac.jp \\ Received 24 December 2010; Accepted 30 March 2011 \\ Academic Editor: Carlos F. Zinola
}

Copyright ( $) 2011$ I. Mogi and K. Watanabe. This is an open access article distributed under the Creative Commons Attribution License, which permits unrestricted use, distribution, and reproduction in any medium, provided the original work is properly cited.

Chiral behavior of magnetoelectrodeposited (MED) $\mathrm{Cu}$ film electrodes was investigated for the electrochemical reactions of amino acids. The $\mathrm{Cu}$ films were electrodeposited under a magnetic field of $5 \mathrm{~T}$ perpendicular to the electrode surface. Such MED Cu films were employed as an electrode, and cyclic voltammograms were measured for the electrochemical reactions of several kinds of amino acids. Chiral behavior was clearly observed as oxidation current difference between the enantiomers of alanine, aspartic acid, and glutamic acid. The MED film electrodes with the thickness of 50 500 nm exhibited such chiral behavior, and their surface morphologies had network structures, which could be induced by the micro-MHD effect.

\section{Introduction}

When a magnetic field is imposed to an electrodeposition process (magnetoelectrodeposition), the Lorentz force acting on the faradaic current causes convection of the electrolytic solution. This is well known as the magnetohydrodynamic (MHD) effect $[1,2]$. The MHD effect leads to spiral structures in the two-dimensional growth of magnetoelectrodeposited (MED) metals [3-6] and conducting polymers [7], and the three-dimensional helical structures in silicate membrane growth [8]. Thus, magnetoelectrodeposition has the potential to produce chiral structures. This is called as magnetoelectrochemical chirality. Control of chirality is one of the most significant subjects in biochemistry, and considerable effort has been devoted in exploring chiral catalysts. If the chiral structures can be formed in nanometer scales on the MED films, such film surfaces would serve as an enantioselective catalyst.

Aogaki proposed the micro-MHD effect in the electrodeposition under the magnetic fields parallel to the faradaic current and perpendicular to the electrode surfaces [9]. In electrodeposition processes, nonequilibrium fluctuation produces a lot of humps on the deposit surfaces. The faradaic current around such humps is not parallel to the magnetic field so that the Lorentz force could cause vortex-like convection in the local areas around the humps. The micro-MHD effect was observed in the circular structures on the MED $\mathrm{Cu}$ film surfaces [9] and the network structures in the Nialumina composite films [10]. The micro-MHD convection breaks the symmetry of mass transfer around the humps, and such symmetry breaking might lead to the formation of chiral structures on the deposit surfaces [11].

We tried the magnetoelectrodeposition of Ag films and employed them as modified electrodes. The MED Ag film electrodes were found to exhibit different oxidation currents between the enantiomers of glucose and cysteine [12, 13]. To demonstrate the universality of magnetoelectrochemical chirality, it is necessary to examine the chiral behaviors of the MED films of various kinds of metals. Copper electrodes exhibit electrocatalytic behaviors for the electro-oxidation of alcohols and amino acids in alkaline aqueous solutions $[14,15]$. The electrocatalytic reactions are sensitive to the surface structure of the electrode through specific adsorption of reactant molecules. If the reactant has chirality, its electrocatalytic reaction could be sensitive to the chirality of the electrode surface. In this paper, we report the magnetoelectrodeposition of $\mathrm{Cu}$ films and their characteristic chiral electrode behaviors for amino acids. 


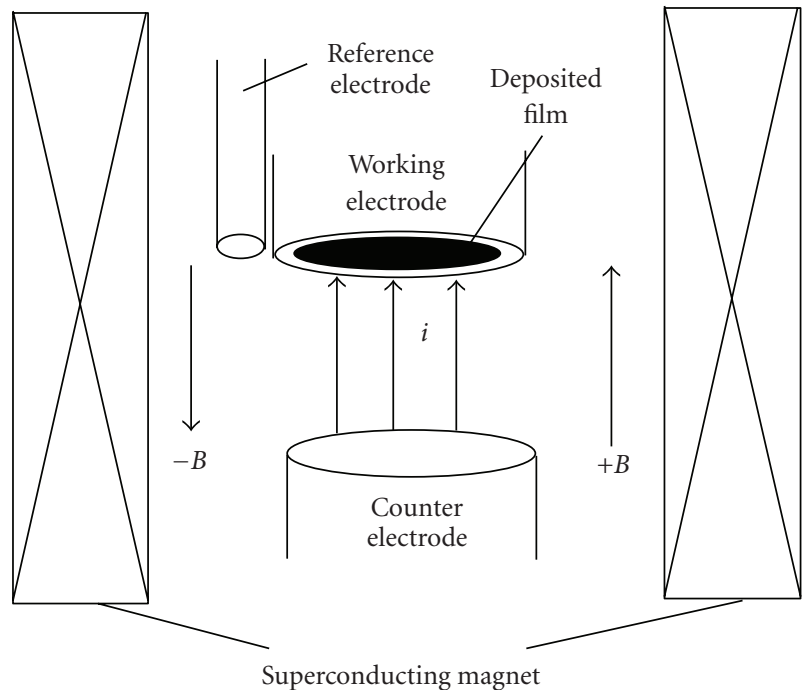

FIGURE 1: Schematic representation of magnetoelectrodeposition in a superconducting magnet. Magnetic fields $B$ are applied to parallel $(+)$ or antiparallel $(-)$ to faradaic currents, and they are perpendicular to the electrode surface.

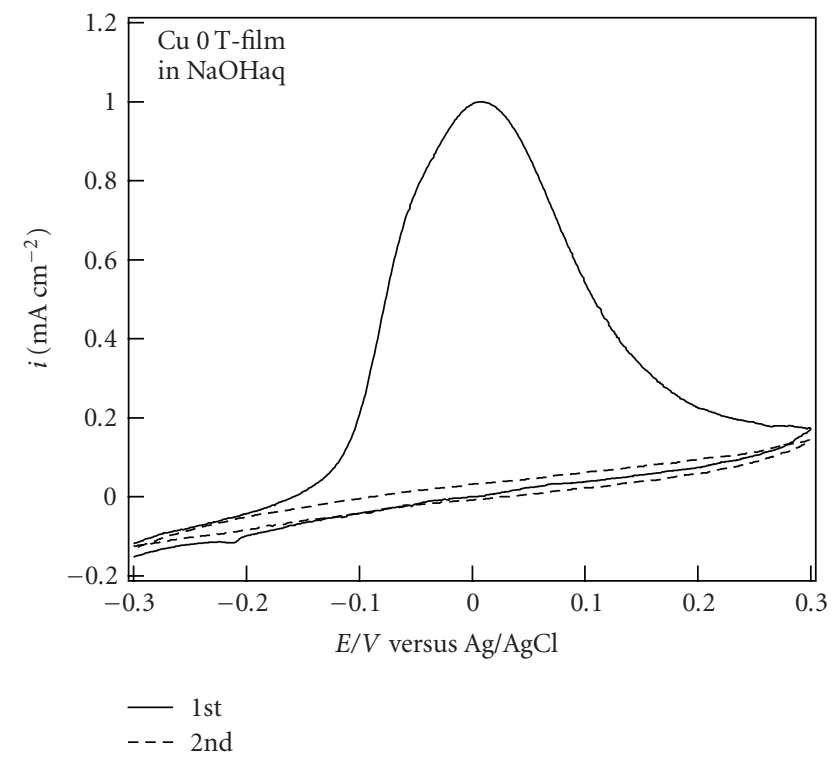

Figure 2: CVs of the $\mathrm{Cu}$ 0T-film electrode in a $0.1 \mathrm{MNaOH}$ aqueous solution. The 1st sweep (solid line) corresponds to the pretreatment of the $\mathrm{Cu}$ film electrode, and the 2nd sweep (dashed line) represents the background current. The potential sweep rate was $10 \mathrm{mV} \mathrm{s}^{-1}$.

\section{Experimental}

All chemicals were reagent grade and were used as received. For electrochemical experiments, a conventional system with the following three electrodes was employed: a polycrystalline Pt disc working electrode with a diameter of $3 \mathrm{~mm}$, a $\mathrm{Cu}$ or Pt plate counter electrode, a $\mathrm{Ag}|\mathrm{AgCl}| \mathrm{NaCl}$ $\left(3 \mathrm{M}\left(\mathrm{M}=\mathrm{mol} \mathrm{dm}^{-3}\right)\right)$ reference electrode. A potentiostat (Princeton Applied Research model 263A) was used for all the electrochemical experiments.

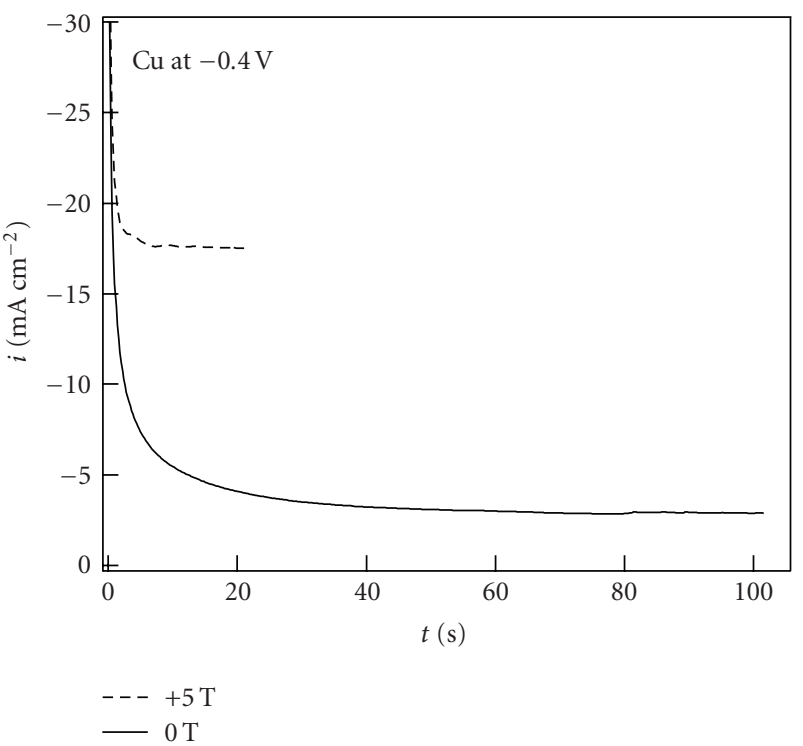

Figure 3: Current-time curves during the $\mathrm{Cu}$ electrodeposition in $0 \mathrm{~T}$ and $+5 \mathrm{~T}$.

$\mathrm{Cu}$ electrodeposition was conducted in a $50 \mathrm{mM} \mathrm{CuSO}_{4}$ aqueous solution containing $0.5 \mathrm{M} \mathrm{H}_{2} \mathrm{SO}_{4}$. The $\mathrm{Cu}$ films were formed on the Pt working electrode by the potentiostatic electrodeposition at a potential of $-0.4 \mathrm{~V}$. The film thickness was controlled by a passing charge, and the thickness could be approximately estimated from the density of $\mathrm{Cu}\left(8.93 \mathrm{~g} \mathrm{~cm}^{-3}\right)$, the electrode area and the passing charge. The usual film thickness was c.a. $150 \mathrm{~nm}$ at the passing charge of $0.4 \mathrm{C} \mathrm{cm}^{-2}$.

Figure 1 shows a schematic representation of the magnetoelectrodeposition. The electrochemical cell was placed at the bore center in a cryocooled superconducting magnet (Sumitomo Heavy Industries Ltd.), which is installed in the High Field Laboratory of Tohoku University and can produce magnetic fields of up to $5 \mathrm{~T}$. An applied magnetic field $B$ was perpendicular to the working electrode surface, and it was parallel $(+B)$ or antiparallel $(-B)$ to the faradaic current. The films prepared at $+5 \mathrm{~T}$ or $-5 \mathrm{~T}$ are called the $+5 \mathrm{~T}$-film or the $-5 \mathrm{~T}$-film, respectively. The temperature within the magnet bore was controlled at $25^{\circ} \mathrm{C}$ by circulating thermoregulated water.

The magnetoelectrodeposited (MED) films were used as modified electrodes, and their chiral properties were examined by measuring cyclic voltammograms (CVs) of the enantiomers of several kinds of amino acids (alanine, aspartic acid, lysine, etc.) in a $0.1 \mathrm{M} \mathrm{NaOH}$ aqueous solution. Because $\mathrm{Cu}$ metal is more easily oxidized than amino acids, the $\mathrm{Cu}$ film electrodes underwent a pretreatment of a potential sweep $(-0.3 \sim 0.3 \mathrm{~V})$ in the $\mathrm{NaOH}$ solution before the CV measurements, as shown in Figure 2 (the solid line). In this potential sweep, the oxidation from $\mathrm{Cu}$ to $\mathrm{Cu}(\mathrm{I})$ occurs around $-0.05 \mathrm{~V}$, and the oxidation from $\mathrm{Cu}(\mathrm{I})$ to $\mathrm{Cu}$ (II) occurs around $0.02 \mathrm{~V}$ [14], forming a stable oxide film of $\mathrm{CuO}$. The oxidation current became very small in the 2 nd potential sweep (the dashed line in Figure 2), indicating that the film surface is covered by $\mathrm{CuO}$ after the pretreatment. 


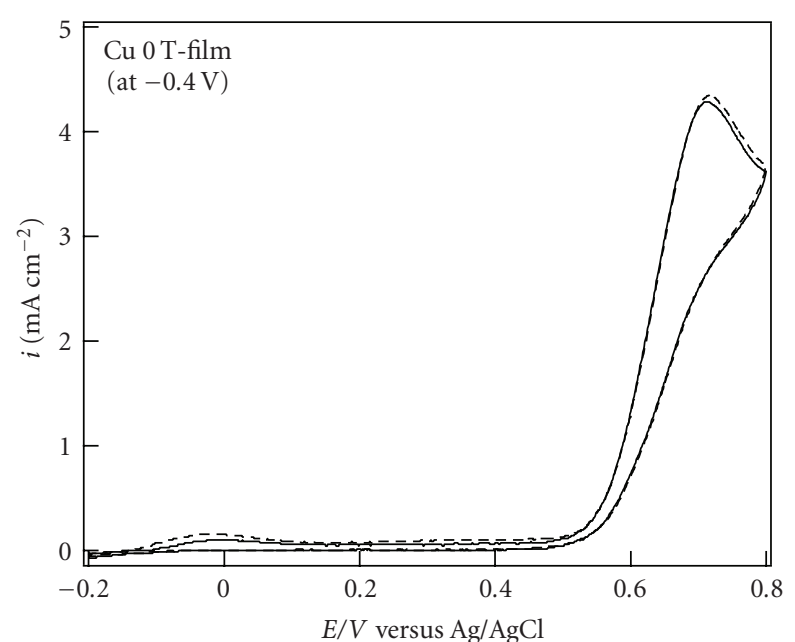

(a)

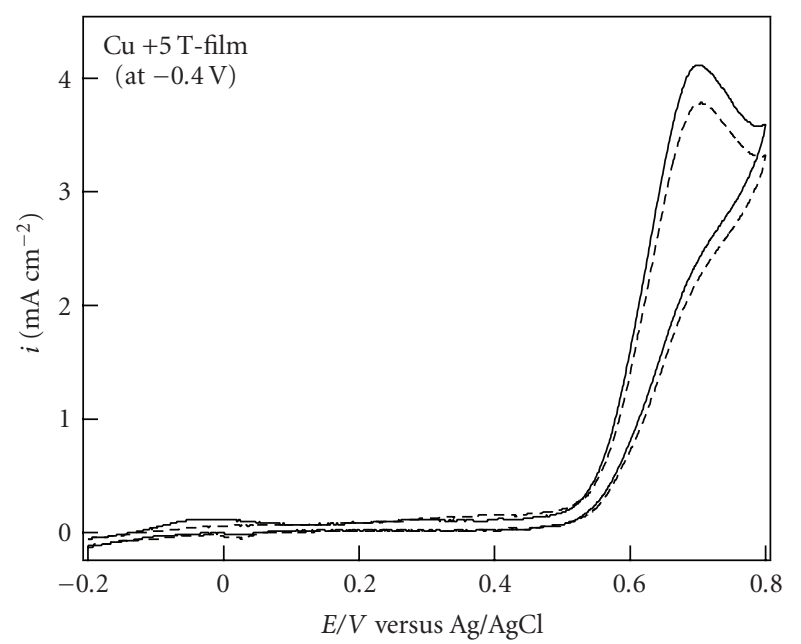

(b)

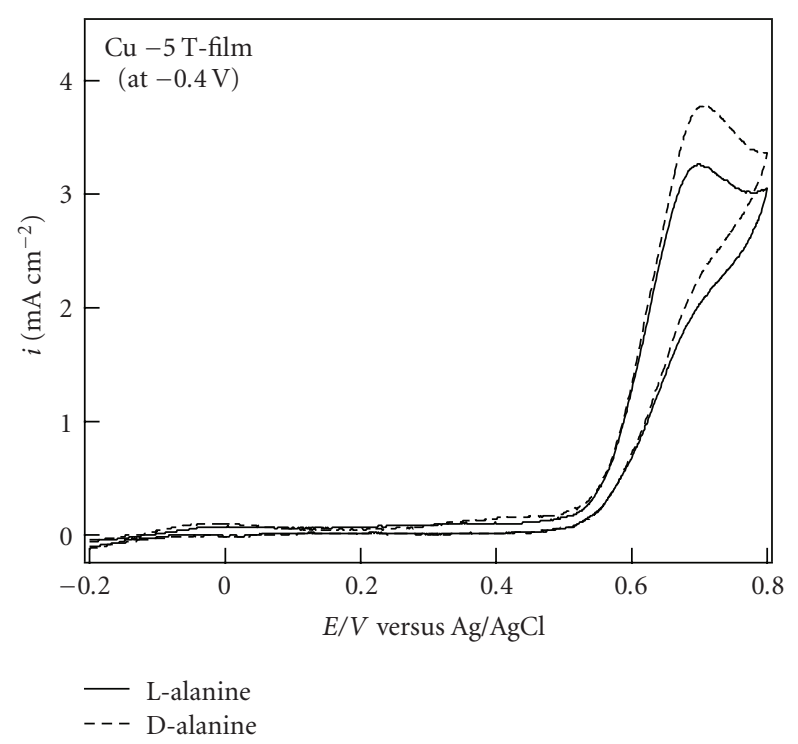

(c)

Figure 4: CVs of $20 \mathrm{mM} \mathrm{L}$ - and D-alanines on the $\mathrm{Cu}$ (a) 0T-film, (b) +5T-film, and (c) $-5 \mathrm{~T}$-film electrodes in a $0.1 \mathrm{M} \mathrm{NaOH}$ aqueous solution. The potential sweep rate was $10 \mathrm{mV} \mathrm{s}^{-1}$.

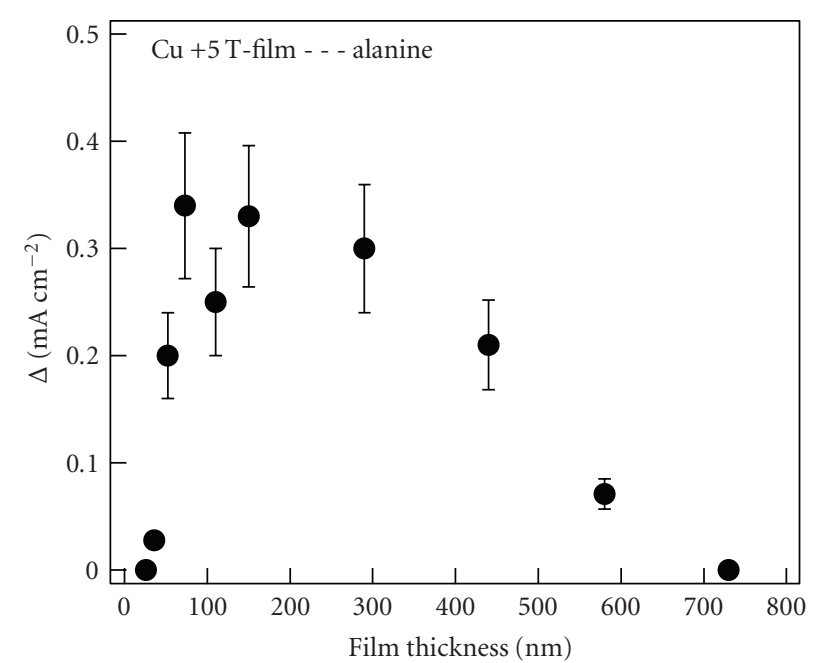

Figure 5: Film thickness dependence of the magnetoelectrochemical chirality. $\Delta$ is the peak current difference between L- and Dalanines in the $\mathrm{CV}$ s on the $\mathrm{Cu}+5 \mathrm{~T}$-film electrodes.

The CVs of amino acids were measured with a potential sweep rate of $10 \mathrm{mV} \mathrm{s}^{-1}$ in the absence of a magnetic field.

\section{Results and Discussion}

Figure 3 shows current-time curves during the Cu electrodeposition in $0 \mathrm{~T}$ and $+5 \mathrm{~T}$. It shows that the magnetic field considerably enhances the electrodeposition currents, even though the applied field is parallel to the faradaic current. The MHD effect is the minimum in, such a configuration, but it never disappears, because the current is not parallel to the magnetic field at the electrode edge and the fluctuated deposit surface. The latter is the micro-MHD effect [9], as mentioned above.

CVs of several amino acids were examined on the MED $\mathrm{Cu}$ film electrodes. Figure 4 shows CVs of $20 \mathrm{mM} \mathrm{L}$ - and Dalanines on the $\mathrm{Cu}$ (a) 0T-film, (b) $+5 \mathrm{~T}$-film, and (c) $-5 \mathrm{~T}$ film electrodes. The oxidation reaction of alanine occurs around $0.7 \mathrm{~V}$, where the amino group $\left(-\mathrm{NH}_{2}\right)$ in the amino acid is oxidized [15]. Both CVs of the enantiomers are almost coincident each other on the 0T-film electrode (Figure 4(a)). On the $+5 \mathrm{~T}$-film electrode, difference between the two CVs is seen in the peak currents. The oxidation current of L-alanine is larger than that of D-alanine (Figure 4(b)). On the contrary, the result is opposite for the MED film prepared under the reversal magnetic field. The oxidation current of D-alanine is larger than that of $\mathrm{L}$ - alanine on the -5 T-film electrode (Figure 4(c)). These results indicate that the magnetoelectrodeposition induces the chirality in the $\mathrm{Cu}$ films and that the MED films possess the ability of enantioselective recognition for $\mathrm{L}$ - and $\mathrm{D}$-alanines. The fact that the chiral behavior depends on the polarity of the magnetic fields indicates that the chirality arises from the Lorentz force and micro-MHD effect.

Similar chiral behavior was observed in the MED Ag film electrode for the glucose oxidation reaction $[12,13]$. Both 


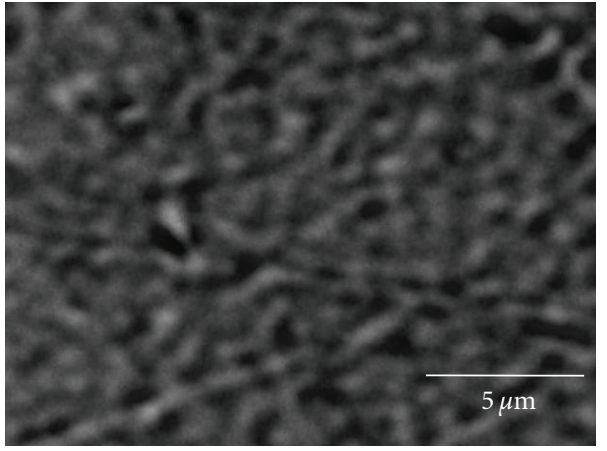

(a)

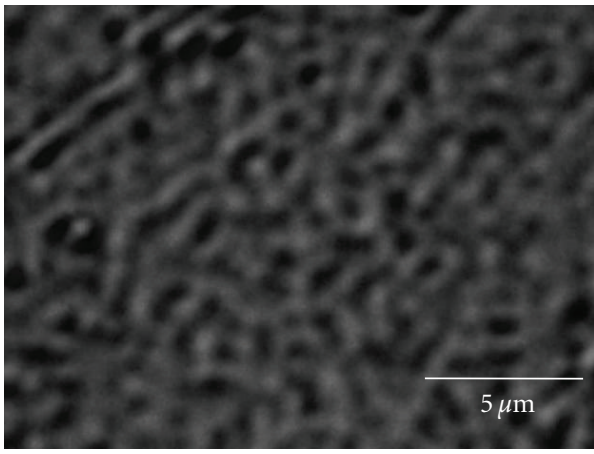

(b)

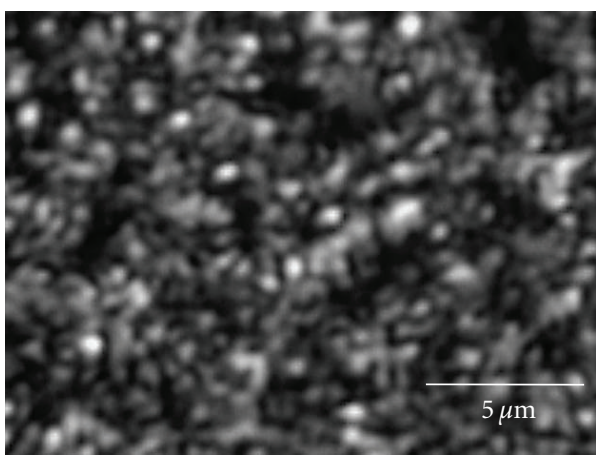

(c)

Figure 6: Microphotographs of the surface morphology of the $\mathrm{Cu}$ +5T-films with the thickness of (a) $150 \mathrm{~nm}$, (b) $440 \mathrm{~nm}$, and (c) $730 \mathrm{~nm}$.

crystal structures of $\mathrm{Ag}$ and $\mathrm{Cu}$ are face-centered cubic (fcc), and generally, such a highly symmetrical crystal has no chirality. However, Attard et al. pointed out that there exist chiral kinks in certain surfaces, for example, $\{643\}$, of the fcc crystal and demonstrated that such a surface exhibits chiral electrode behaviors for the oxidation of D- and L-glucoses [16]. Similarly, they proposed that corner kinks of crystals could be a chiral site [17]. These ideas might be crucial for considering the chiral sites on the MED film surfaces.

The film thickness dependence of the chiral behavior of the $+5 \mathrm{~T}$-film was examined for the electrochemical reaction of alanine. Figure 5 shows the oxidation current difference $\Delta\left(=i_{p}(\mathrm{~L})-i_{p}(\mathrm{D})\right)$ between the enantiomers against the film thickness, where $i_{p}(\mathrm{~L})$ and $i_{p}(\mathrm{D})$ represent peak currents

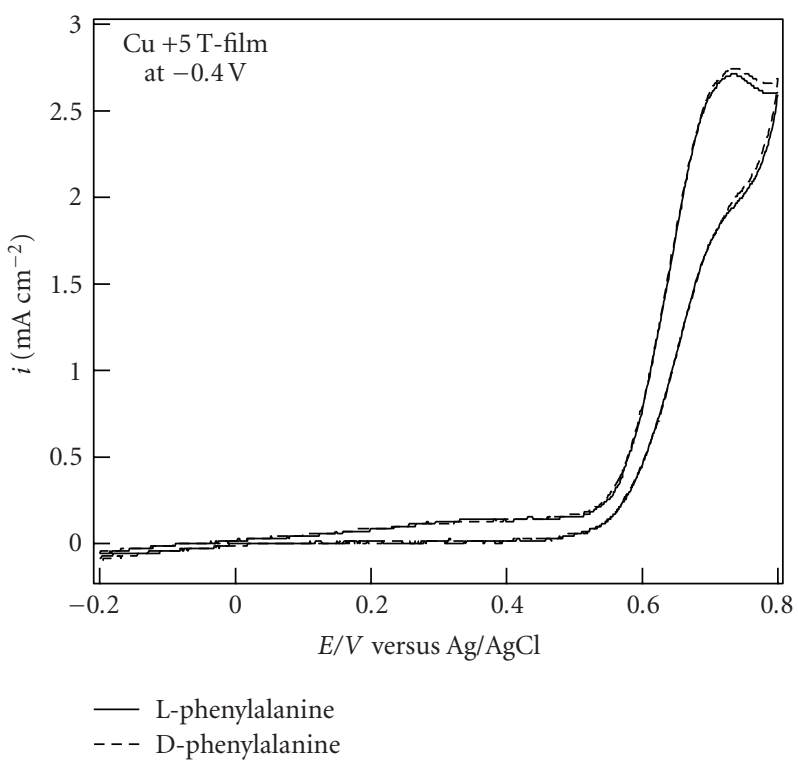

Figure 7: CVs of $20 \mathrm{mM} \mathrm{L}$ - and D-phenylalanines on the $\mathrm{Cu}+5 \mathrm{~T}$ film electrodes in a $0.1 \mathrm{M} \mathrm{NaOH}$ aqueous solution. The potential sweep rate was $10 \mathrm{mV} \mathrm{s}^{-1}$.

of the CVs of L- and D-alanines, respectively. This figure shows that the chiral behavior appears in the thickness range of $50 \sim 500 \mathrm{~nm}$. Figure 6 shows the surface morphologies of the $\mathrm{Cu}+5 \mathrm{~T}$-films with the thickness of (a) $150 \mathrm{~nm}$, (b) $440 \mathrm{~nm}$, and (c) $730 \mathrm{~nm}$, where the photomicrographs were taken just after the magnetoelectrodeposition without the pretreatment. The films (a) and (b) exhibit the chiral behavior for alanine, and their surface morphologies possess network structures, which could be caused by the microMHD effect. Similar network structures were observed in the MED Ni- $\mathrm{Al}_{2} \mathrm{O}_{3}$ composite films [9] and magnetocorrosion of $\mathrm{Zn}$ single crystals [18]. On the contrary, the film (c) shows no chiral behavior, and the surface morphology is random aggregates of particles with a size of $\sim 1 \mu \mathrm{m}$. These results imply that the micro-MHD effect induces the selforganization of convections and deposit structures in the film formation and that random fluctuation becomes dominant with increasing film thickness. Such self-organization seems to be significant for the formation of chiral surfaces.

We examined chiral response of the MED $\mathrm{Cu}$ film electrodes for several kinds of amino acids. Figure 7 shows CVs of $20 \mathrm{mM}$ phenylalanine on the $+5 \mathrm{~T}$-film electrode. The two CV curves are coincident each other, thus the MED film exhibits no chiral recognition for phenylalanine. This is contrary to the results of alanine, as shown in Figure 4. When amino acids are represented as $\mathrm{R}-\mathrm{CH}(\mathrm{COOH})\left(\mathrm{NH}_{2}\right)$, $\mathrm{R}$ is a methyl group $\left(-\mathrm{CH}_{3}\right)$ in alanine, and it is a benzyl group $\left(-\mathrm{CH}_{2} \mathrm{C}_{6} \mathrm{H}_{5}\right)$ in phenylalanine. The benzyl group is more bulky than the methyl group, and such a steric effect could prevent the specific adsorption at the chiral sites on the electrode surface, leading to the lack of chiral recognition.

Figure 8 shows CVs of $20 \mathrm{mM}$ (a) aspartic acid, (b) glutamic acid, (c) lysine, and (d) arginine on the +5 T-film electrode. While the MED film exhibits the chiral recognition 


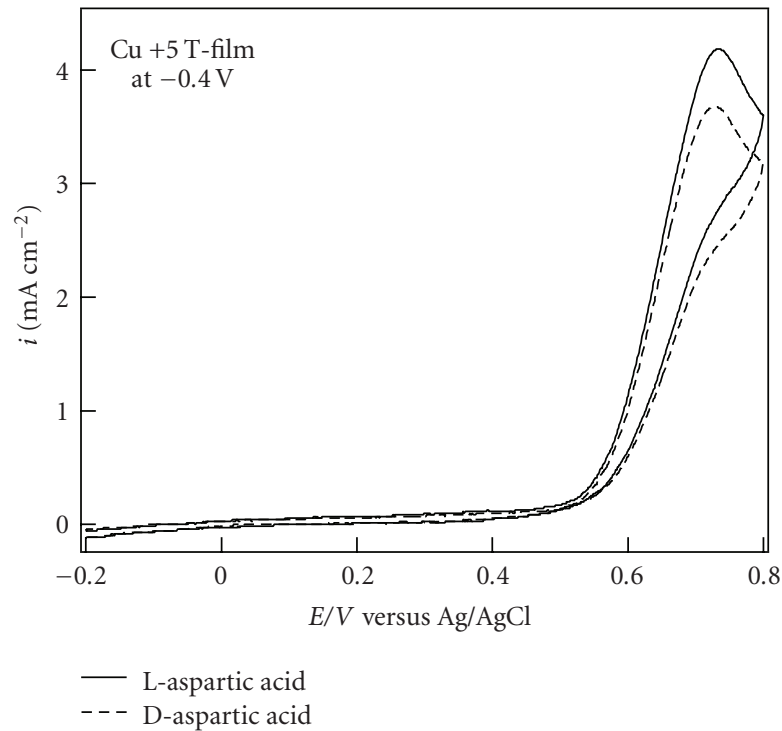

(a)

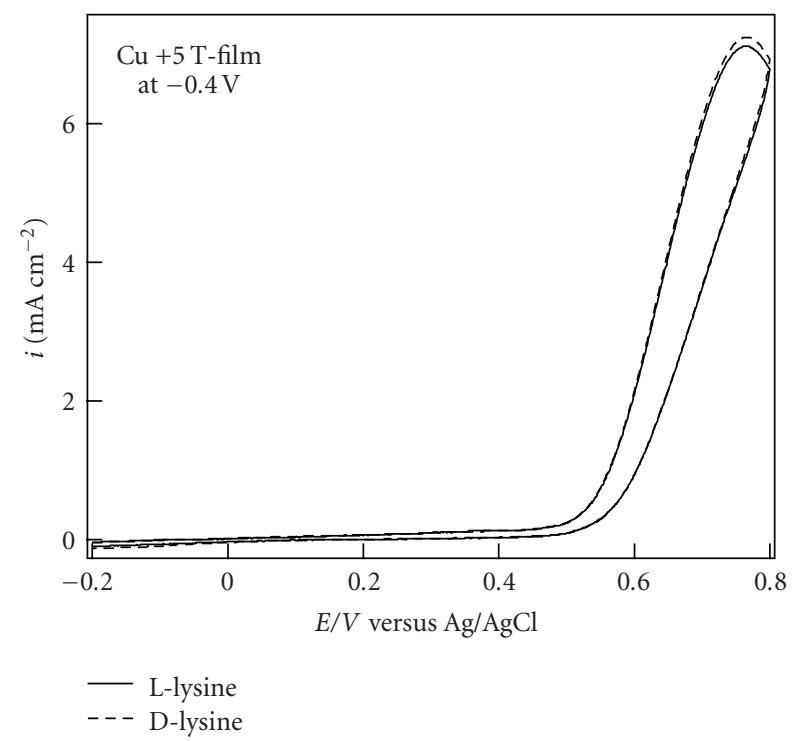

(c)

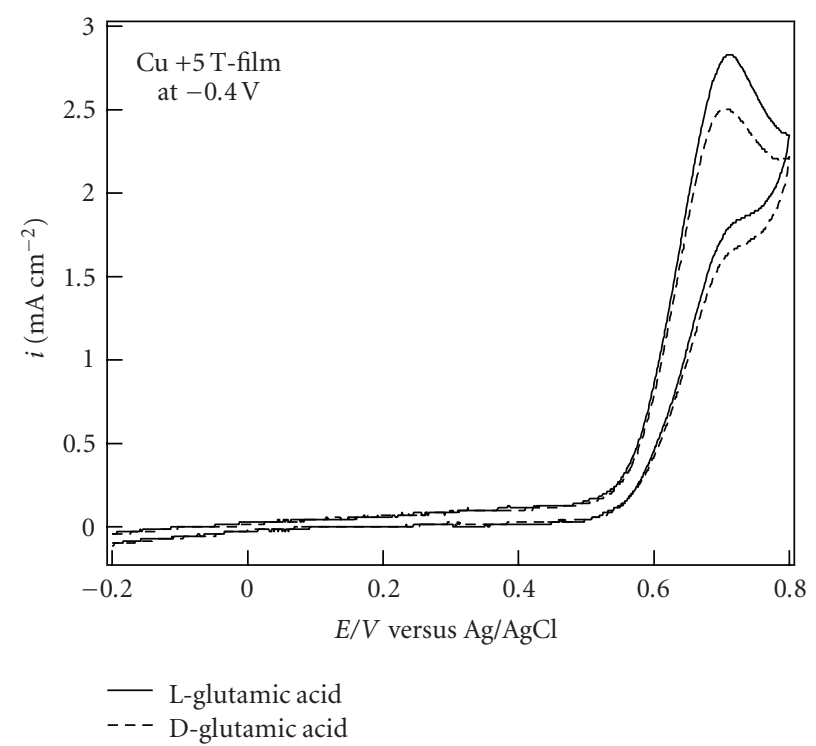

(b)

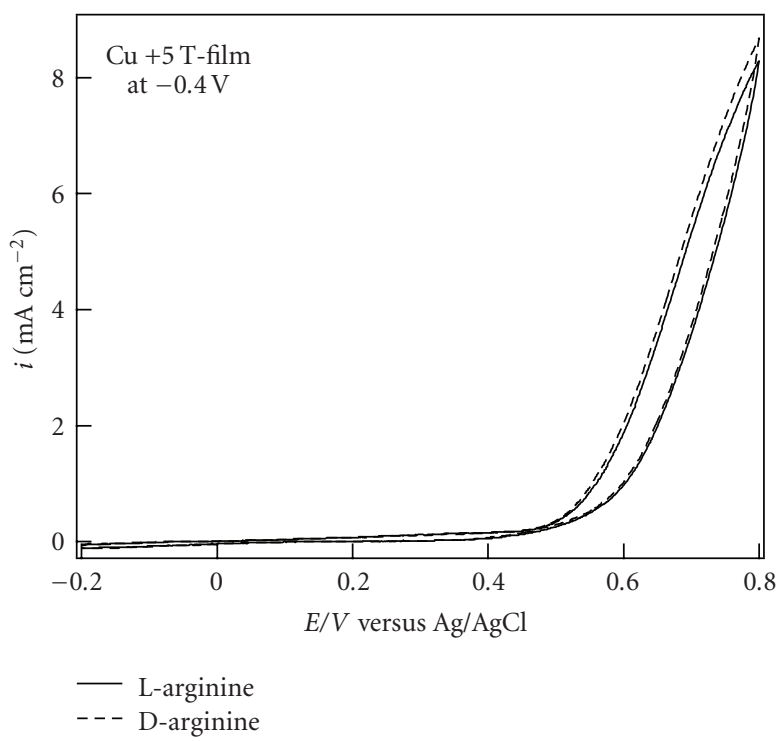

(d)

Figure 8: CVs of the enantiomers of (a) aspartic acid, (b) glutamic acid, (c) lysine, and (d) arginine on the Cu +5 T-film electrodes in a $0.1 \mathrm{M} \mathrm{NaOH}$ aqueous solution. The potential sweep rate was $10 \mathrm{mV} \mathrm{s}^{-1}$.

for aspartic and glutamic acids, it shows no chiral behavior for lysine and arginine. The molecular structures of R-groups are clearly different between the formers and the latters. The $\mathrm{R}$-groups of aspartic and glutamic acids involve a carboxyl group $(-\mathrm{COOH})$, on the contrary, those of lysine and arginine involve an amino group $\left(-\mathrm{NH}_{2}\right)$. These facts suggest that the carboxyl group promotes the specific adsorption on the electrode surface, leading to the chiral recognition. On the other hands, the amino group participates in the electrode reaction and contributes to the large oxidation currents, as shown in Figures 8(c) and 8(d). This seems to be crucial for the lack of chiral recognition for lysine and arginine.
There remains interesting open issues where the chiral site is on the MED film surface, why such a chiral site can survive after the surface oxidation in the pretreatment process, and how the chiral site recognizes the molecular chirality of amino acids. To elucidate these issues in future studies leads to a design of electrocatalytic chiral surface using the magnetoelectrodeposition technique.

\section{Conclusion}

We have shown that the MED Cu film electrodes exhibit chiral behaviors for the oxidation of the enantiomers of alanine, 
aspartic acid, and glutamic acid and that such magnetoelectrochemical chirality depends on the film thickness. Taking account of our previous results of the chirality of the MED Ag films [12, 13], the observation of the chirality in the MED $\mathrm{Cu}$ films implies that the magnetoelectrochemical chirality is a universal phenomenon in the magnetoelectrodeposition of metals. It is noteworthy that the magnetoelectrodeposition is able to provide chiral surfaces without any chiral chemicals, and this novel technique would be applicable to new design of chiral catalysts and sensors.

\section{Acknowledgment}

The magnetoelectrochemical experiments were performed in the High Field Laboratory for Superconducting Materials, IMR Tohoku University.

\section{References}

[1] R. Aogaki, K. Fueki, and T. Mukaibo, "Application of magnetohydrodynamic effect to the analysis of electrochemical reactions-1," Denki Kagaku, vol. 43, no. 9, pp. 504-508, 1975.

[2] T. Z. Fahidy, "Magnetoelectrolysis," Journal of Applied Electrochemistry, vol. 13, no. 5, pp. 553-563, 1983.

[3] I. Mogi, S. Okubo, and Y. Nakagawa, "Dense radial growth of silver metal leaves in a high magnetic field," Journal of the Physical Society of Japan, vol. 60, no. 10, pp. 3200-3202, 1991.

[4] I. Mogi, M. Kamiko, and S. Okubo, "Magnetic field effects on fractal morphology in electrochemical deposition," Physica B, vol. 211, no. 1-4, pp. 319-322, 1995.

[5] J. M. D. Coey, G. Hinds, and M. E. G. Lyons, "Magnetic-field effects on fractal electrodeposits," Europhysics Letters, vol. 47, no. 2, pp. 267-272, 1999.

[6] V. Heresanu, R. Ballou, and P. Molho, "Electrochemical growth of $\mathrm{Zn}$ and $\mathrm{Fe}$ arborescence under normal magnetic field," Magnetohydrodynamics, vol. 39, no. 4, pp. 461-469, 2003.

[7] I. Mogi and M. Kamiko, "Pattern formation in magnetoelectropolymerization of pyrrole," Denki Kagaku, vol. 64, no. 7, pp. 842-844, 1996.

[8] W. Duan, S. Kitamura, I. Uechi, A. Katsuki, and Y. Tanimoto, "Three-dimensional morphological chirality induction using high magnetic fields in membrane tubes prepared by a silicate garden reaction," Journal of Physical Chemistry B, vol. 109, no. 28, pp. 13445-13450, 2005.

[9] R. Aogaki, "Micro-MHD effect on electrodeposition in the vertical magnetic field," Magnetohydrodynamics, vol. 39, no. 4, pp. 453-460, 2003.

[10] T. Yamada and S. Asai, "Distribution control of dispersed particles in a film fabricated by composite plating method using a high magnetic field," Journal of the Japan Institute of Metals, vol. 69, no. 2, pp. 257-262, 2005.

[11] R. Aogaki, R. Morimoto, A. Sugiyama, and M. Asanuma, "Origin of chirality in magnetoelectrodeposition," in Proceedings of the 6th International Conference on Electromagnetic Processing of Materials, pp. 439-442, Dresden, Germany, 2009.

[12] I. Mogi and K. Watanabe, "Chiral electrode behavior of magnetoelectrodeposited silver films," ISIJ International, vol. 47, no. 4, pp. 585-587, 2007.

[13] I. Mogi and K. Watanabe, "Magnetoelectrochemical chirality in Ag electrodeposition," Journal of Chemistry and Chemical Engineering, vol. 4, no. 11, pp. 16-22, 2010.
[14] N. Torto, T. Ruzgas, and L. Gorton, "Electrochemical oxidation of mono- and disaccharides at fresh as well as oxidized copper electrodes in alkaline media," Journal of Electroanalytical Chemistry, vol. 464, no. 2, pp. 252-258, 1999.

[15] P. Luo, F. Zhang, and R. P. Baldwin, "Constant potential amperometric detection of underivatized amino acids and peptides at a copper electrode," Analytical Chemistry, vol. 63, no. 17, pp. 1702-1707, 1991.

[16] G. A. Attard, C. Harris, E. Herrero, and J. Feliu, "The influence of anions and kink structure on the enantioselective electrooxidation of glucose," Faraday Discussions, vol. 121, pp. 253266, 2002.

[17] G. A. Attard, A. Ahmadi, D. J. Jenkins et al., "The characterisation of supported platinum nanoparticles on carbon used for enantioselective hydrogenation," ChemPhysChem, vol. 4, no. 2, pp. 123-130, 2003.

[18] K. Shinohara, K. Hashimoto, and R. Aogaki, "Hexagonal corrosion pattern upon cleavage of a zinc single crystal under a vertical high magnetic field," Chemistry Letters, vol. 31, no. 8, pp. 778-779, 2002. 


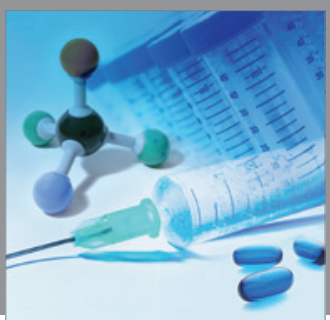

International Journal of

Medicinal Chemistry

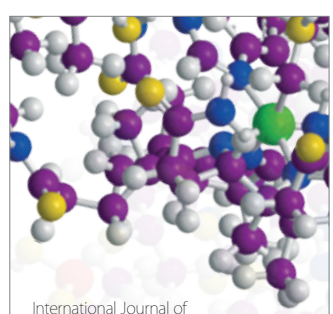

Carbohydrate Chemistry

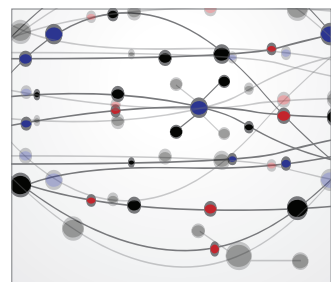

The Scientific World Journal
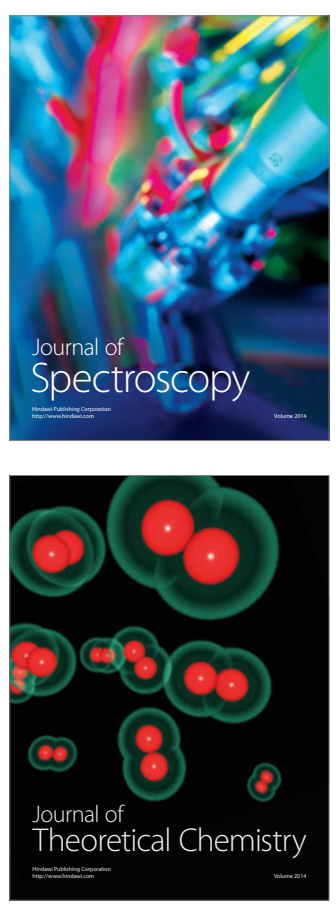
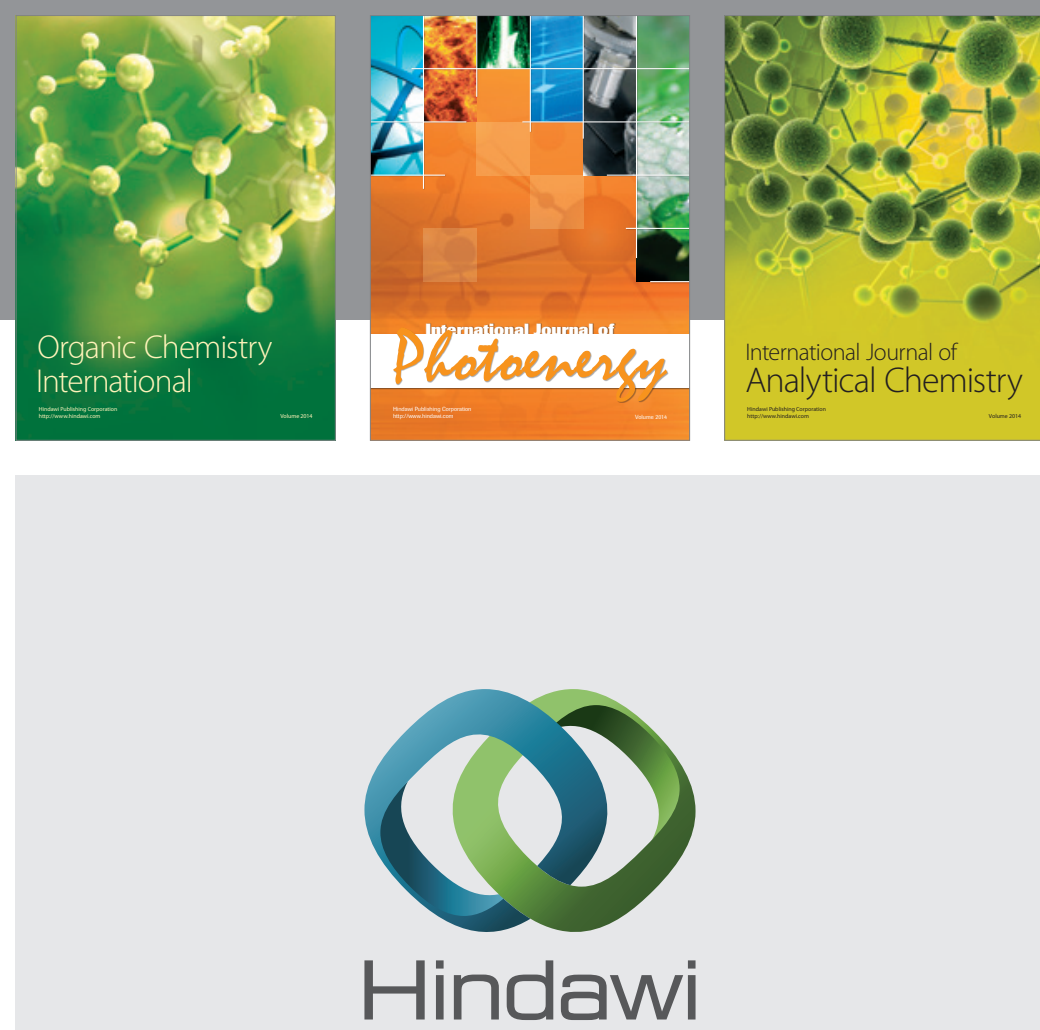

Submit your manuscripts at

http://www.hindawi.com
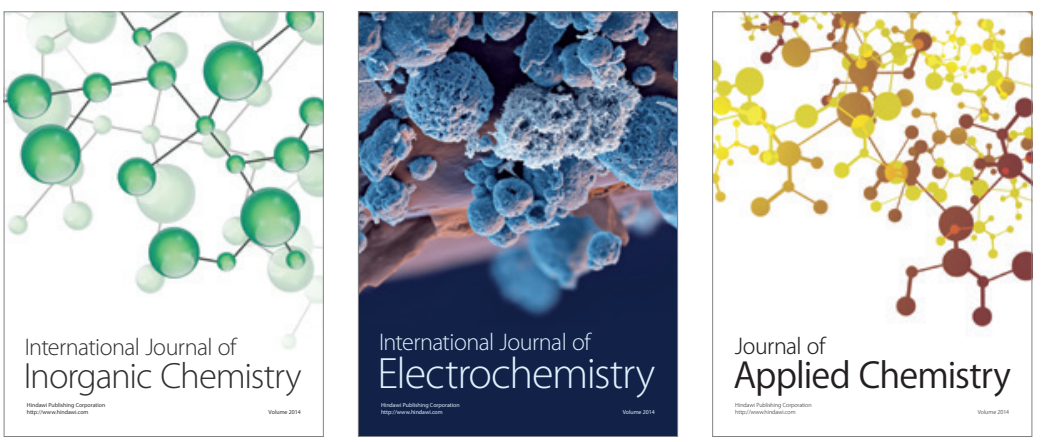

Journal of

Applied Chemistry
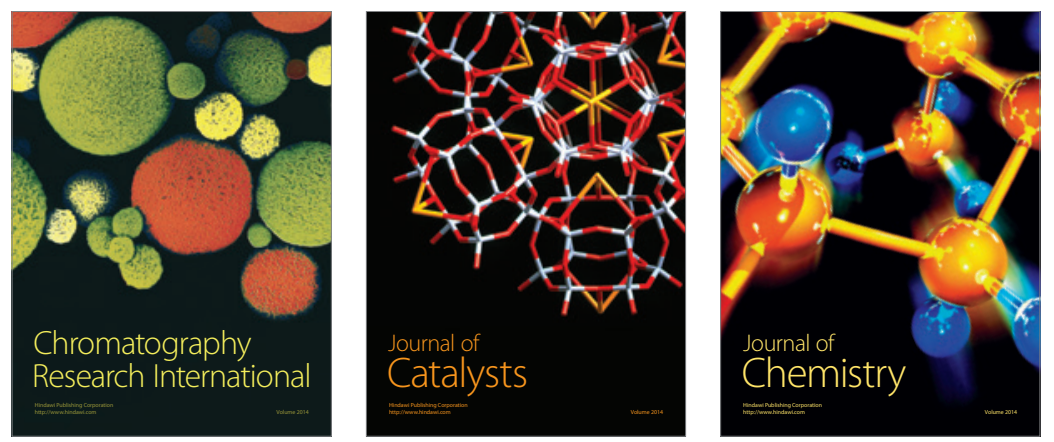
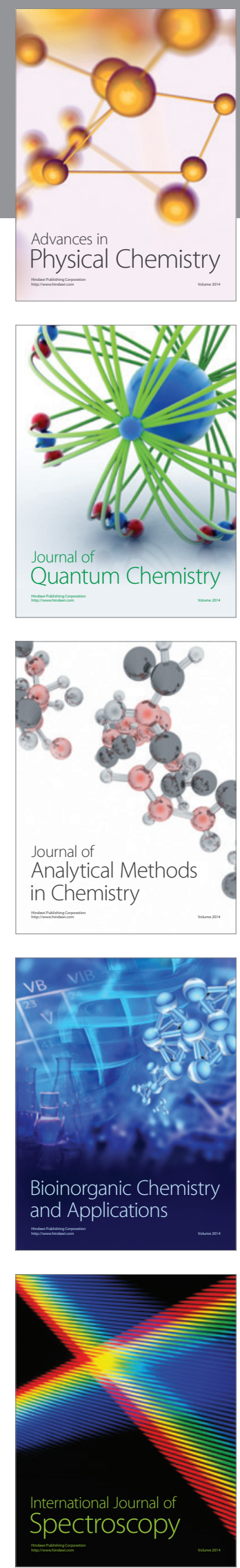